\title{
On the Solutions of a Stefan Problem with Variable Latent Heat
}

\author{
Bougoffa Lazhar \\ Department of Mathematics, Faculty of Science, Al Imam Mohammad Ibn Saud Islamic University (IMSIU), \\ P.O. Box 90950, Riyadh 11623, Saudi Arabia
}

Correspondence should be addressed to Bougoffa Lazhar; bougoffa@hotmail.com

Received 17 April 2014; Accepted 3 July 2014; Published 13 July 2014

Academic Editor: Ashraf M. Zenkour

Copyright (C) 2014 Bougoffa Lazhar. This is an open access article distributed under the Creative Commons Attribution License, which permits unrestricted use, distribution, and reproduction in any medium, provided the original work is properly cited.

This paper is concerned with a reliable treatment of the Stephan problem with variable latent heat. The power series solutions have been presented. The method is examined for computational efficiency and accuracy. Also, an analytical solution based on a similarity variable is presented in the case when the Dirichlet boundary condition at the water-ice interface depends on time.

\section{Introduction}

The solution of the Stefan problem with variable latent heat consists of finding $u$ and the moving melt interface $s$ such that

$$
\frac{\partial u}{\partial t}=\frac{\partial^{2} u}{\partial x^{2}}, \quad 0 \leq x \leq s(t),
$$

which is the governing equation, subject to the following Neumann boundary condition at the left end of the domain describing the inlet heat flux:

$$
-\frac{\partial u}{\partial x}(t, 0)=h(t), \quad t>0,
$$

the Dirichlet boundary condition at the water-ice interface:

$$
u(t, s(t))=u^{*}, \quad t>0,
$$

the Stefan condition:

$$
s \frac{d s}{d t}=-\frac{\partial u}{\partial x}(t, s(t)), \quad s(0)=b, t>0,
$$

and the initial temperature distribution:

$$
u(0, x)=\varphi(x), \quad 0<x<s(0)=b .
$$

On comparison of this condition with the one-phase Stefan condition, we observe that the latent heat term $s$ is not a constant but, rather, a linear function of position [1].
Problems such as this have been treated under the name of Stefan problems with variable latent heat [1-3].

An analytical solution for this Stefan problem is presented in [1], by introducing the similarity variables $\xi=x / \sqrt{t}$ and seeking the solution in the form $u(t, x)=F(\xi)$ with $F$ being an unknown function. Accordingly, it is natural that the interface location $s(t)$ should be proportional to $\sqrt{t}$; that is, $s(t)=A \sqrt{t}$, where $A$ is a constant.

The purpose of this paper is to apply the Adomian decomposition method [4-25] to find the solution of (1), (2), and (5), that is, the temperature distribution of the water $u(t, x)$, and then use a direct method to determine the position of the ice-water interface as a function of time. Also, an analytical solution based on a similarity variable is presented in the case when the Dirichlet boundary condition at the water-ice interface depends on time of the form $u^{*}(t)=$ $\alpha \sqrt{t}$, where $\alpha$ is a constant.

\section{The Adomian Decomposition Method}

Based on the Adomian decomposition method, we write (1) in Adomian's operator-theoretic notation as

$$
L_{x x} u=\frac{\partial u}{\partial t},
$$

where

$$
L_{x x}=\frac{\partial^{2}}{\partial x^{2}} .
$$


We conveniently define the inverse linear operator as

$$
L_{x x}^{-1}(\cdot)=\iint_{0}^{x}(\cdot) d x d x
$$

Applying the inverse linear operator $L_{x x}^{-1}$ to (6) and taking into account that $(\partial u / \partial x)(t, 0)=-h(x)$, we obtain

$$
u(t, x)=A(t)-h(t) x+\iint_{0}^{x} \frac{\partial u}{\partial t} d x d x
$$

where the unknown boundary condition $A(t)=u(t, 0)$ will be determined.

Define the solution $u(t, x)$ by an infinite series of components in the form

$$
u(t, x)=\sum_{n=0}^{\infty} u_{n}(t, x)
$$

Consequently, the components $u_{n}$ can be elegantly determined by setting the recursion scheme

$$
\begin{gathered}
u_{0}=A(t)-h(t) x \\
u_{n+1}=\iint_{0}^{x} \frac{\partial u_{n}}{\partial t} d x d x, \quad n \geq 0,
\end{gathered}
$$

for the complete determination of these components. In view of (11), the components $u_{0}(t, x), u_{1}(t, x), u_{2}(t, x), \ldots$ are immediately determined as

$$
\begin{gathered}
u_{0}=A(t)-h(t) x \\
u_{1}=A^{\prime}(t) \frac{x^{2}}{2 !}-h^{\prime}(t) \frac{x^{3}}{3 !} \\
u_{2}=A^{\prime \prime}(t) \frac{x^{4}}{4 !}-h^{\prime \prime}(t) \frac{x^{5}}{5 !}
\end{gathered}
$$

Consequently, the solution is readily found to be

$$
u(t, x)=\sum_{n=0}^{\infty} A^{(n)}(t) \frac{x^{2 n}}{(2 n) !}-\sum_{n=0}^{\infty} h^{(n)}(t) \frac{x^{2 n+1}}{(2 n+1) !}
$$

obtained by substituting (12) into (10).

We remark here that the unknown boundary condition $A(t)$ can be easily determined by using the initial condition equation (5). Substituting $t=0$ into (13) and using the Taylor expansion of $\varphi(x)$ lead to

$$
\sum_{n=0}^{\infty} A^{(n)}(0) \frac{x^{2 n}}{(2 n) !}-\sum_{n=0}^{\infty} h^{(n)}(0) \frac{x^{2 n+1}}{(2 n+1) !}=\sum_{n=0}^{\infty} \varphi^{(n)}(0) \frac{x^{n}}{n !} .
$$

Equating the coefficients of like power of $x$ in both sides of (14) and taking into account that the compatibility conditions

$$
h^{(n)}(0)=-\varphi^{(2 n+1)}(0), \quad n \geq 0,
$$

yield

$$
A^{(n)}(0)=\varphi^{(2 n)}(0), \quad n \geq 0
$$

Thus

$$
A(t)=\sum_{n=0}^{\infty} \varphi^{(2 n)}(0) \frac{t^{n}}{(n) !} .
$$

Accordingly, the solution equation (13) is completely determined by defining the function $A(t)$.

Once the function $u(t, x)$ is obtained, we can rewrite the Stefan condition equation (4) in terms of the known function $u(t, x)$ including the Dirichlet and Neumann boundary conditions. For this, integrating (1) with respect to $x$ from 0 to $x$ and taking into account that $-(\partial u / \partial x)(t, 0)=h(t)$, we obtain

$$
\frac{\partial u}{\partial x}=-h(t)+\int_{0}^{x} \frac{\partial u}{\partial t} d x .
$$

Thus (4) can be replaced by

$$
s \frac{d s}{d t}=h(t)-\int_{0}^{s(t)} \frac{\partial u}{\partial t} d x, \quad s(0)=b, t>0 .
$$

Using the following Leibniz rule for differentiation under the integral sign:

$$
\frac{d}{d t} \int_{0}^{s(t)} u(t, x) d x=\int_{0}^{s(t)} \frac{\partial u}{\partial t} d x+u(t, s(t)) \frac{d s}{d t},
$$

and taking into account that $u(t, s(t))=u^{*}$, we obtain

$$
\int_{0}^{s(t)} \frac{\partial u}{\partial t} d x=\frac{d}{d t} \int_{0}^{s(t)} u(t, x) d x-u^{*} \frac{d s}{d t} .
$$

Substituting (21) into (19), we obtain

$$
s \frac{d s}{d t}-u^{*} \frac{d s}{d t}+\frac{d}{d t} \int_{0}^{s(t)} u(t, x) d x=h(t) .
$$

Since $\left(d s^{2} / d t\right)=2 s(d s / d t)$, we have

$$
\frac{d}{d t}\left[s^{2}-2 u^{*} s+2 \int_{0}^{s(t)} u(t, x) d x\right]=2 h(t) .
$$

Applying the inverse linear operator $L_{t}^{-1}(\cdot)=\int_{0}^{t}(\cdot) d t$ to $(23)$, we obtain

$$
\begin{aligned}
s^{2}-2 u^{*} s+2 \int_{0}^{s(t)} u(t, x) d x= & b^{2}-2 b u^{*} \\
& +2 \int_{0}^{t} h(t) d t \\
& +2 \int_{0}^{b} u(0, x) d x
\end{aligned}
$$

It follows that

$$
\begin{aligned}
s^{2}-2 u^{*} s+2 \int_{0}^{s(t)} u(t, x) d x= & b^{2}-2 b u^{*} \\
& +2 \int_{0}^{t} h(t) d t \\
& +2 \int_{0}^{b} \varphi(x) d x .
\end{aligned}
$$


Substituting the solution equation (13) of the heat equation into (25), we obtain

$$
\begin{aligned}
& s^{2}-2 u^{*} s \\
& \quad+2 \int_{0}^{s(t)}\left[\sum_{n=0}^{\infty} A^{(n)}(t) \frac{x^{2 n}}{(2 n) !}-\sum_{n=0}^{\infty} h^{(n)}(t) \frac{x^{2 n+1}}{(2 n+1) !}\right] d x \\
& =g(t),
\end{aligned}
$$

where

$$
g(t)=b^{2}-2 b u^{*}+2 \int_{0}^{t} h(t) d t+2 \int_{0}^{b} \varphi(x) d x .
$$

Thus

$$
s^{2}-2 u^{*} s+F(t, s(t))=g(t),
$$

where

$$
F(t, s(t))=2\left[\sum_{n=0}^{\infty} A^{(n)}(t) \frac{s^{2 n+1}}{(2 n+1) !}-\sum_{n=0}^{\infty} h^{(n)}(t) \frac{s^{2 n+2}}{(2 n+2) !}\right] .
$$

We now can determine the shoreline $s(t)$ with time by solving the nonlinear equation (28). In order to demonstrate the feasibility and efficiency of this method, we consider the following case:

$$
h(t)=e^{-t} \text {. }
$$

If we choose $\varphi(x)=e^{x}$, then a simple calculation leads to $A(t)=e^{t}$, and (28) becomes

$$
s^{2}-2 u^{*} s+e^{t} \sum_{n=0}^{\infty} \frac{s^{n}}{n !}=g(t),
$$

where $F(t, s(t))=e^{t+s}$.

Let us write $s(t)$ and $g(t)$ in series forms $s(t)=\sum_{n=0}^{\infty} s_{n} t^{n}$ and $g(t)=\sum_{n=0}^{\infty} g_{n} t^{n}$. Thus

$$
s(t)=\sum_{n=0}^{\infty} s_{n} t^{n}=\sum_{n=0}^{\infty} \frac{1}{n !}\left[s_{n} t^{n}\right]^{n} .
$$

To compute $\left[s_{n} t^{n}\right]^{n}$ we need the following theorem [4].

Theorem 1. If $u=\sum_{n=0}^{\infty} a_{n} t^{n}$ and $F(u)=\sum_{n=0}^{\infty} b_{n} t^{n}$ are convergent, then $F(u)=\sum_{n=0}^{\infty} \beta_{n} t^{n}$ is convergent, where

$$
\begin{gathered}
\beta_{0}=b_{0}+\sum_{n=0}^{\infty} b_{n} A_{0}^{n}\left(a_{0}\right), \quad \beta_{1}=\sum_{n=0}^{\infty} b_{n} A_{1}^{n}\left(a_{0}, a_{1}\right), \\
\beta_{2}=\sum_{n=0}^{\infty} b_{n} A_{2}^{n}\left(a_{0}, a_{1}, a_{2}\right), \ldots,
\end{gathered}
$$

and $A_{m}^{n}\left(a_{0}, a_{1}, \ldots, a_{m}\right)$ are the Adomian polynomials.
Using this theorem with the given formula $b_{n}=1 / n$ !, we see that

$$
\begin{gathered}
s(t)=\sum_{n=0}^{\infty} s_{n} t^{n}=\sum_{n=0}^{\infty} \beta_{n} t^{n} \\
\beta_{0}=\sum_{n=0}^{\infty} \frac{1}{n !} s_{0}^{n}, \quad \beta_{1}=\sum_{n=1}^{\infty} \frac{1}{n !}\left(n s_{1} s_{0}^{n-1}\right), \ldots
\end{gathered}
$$

Consequently, we obtain the recurrence relations for the coefficients

$$
c_{n}-2 u^{*} s_{n}+\gamma_{n}=g_{n}, \quad n \geq 0,
$$

where $c_{n}=\sum_{k=0}^{n} s_{k} s_{n-k}$ and $\gamma_{n}=\sum_{k=0}^{n}(1 / k !) \beta_{n-k}$.

This solution, of course, is

$$
s(t)=\sum_{n=0}^{\infty} s_{n} t^{n}
$$

\section{Similarity Solution}

We begin our approach by introducing the similarity variable

$$
\xi=\frac{x}{\sqrt{t}}
$$

and look for solutions of (1) in the form

$$
u(t, x)=t^{p} \mu(\xi(t, x)),
$$

where the number $p$ and the function $\mu$ are to be determined. Substituting (39) into (1) we find that

$$
t^{p-1}\left(p \mu-\frac{d^{2} \mu}{d \xi^{2}}-\frac{\xi}{2} \frac{d \mu}{d \xi}\right)=0
$$

and so

$$
\frac{d^{2} \mu}{d \xi^{2}}+\frac{\xi}{2} \frac{d \mu}{d \xi}=p \mu
$$

This is also difficult to solve for arbitrary values of $p$ but for special values we can do something. Define $F(\xi)=\mu e^{\xi^{4} / 4}$, and thus (41) can be transformed into

$$
\frac{d^{2} F}{d \xi^{2}}-\frac{\xi}{2} \frac{d F}{d \xi}=\frac{2 p+1}{2} F
$$

This has the general solution

$$
F(\xi)=C \xi e^{\xi^{2} / 4}+D\left[-\sqrt{\pi} \xi e^{-\xi^{2} / 4} \operatorname{erf}\left(\frac{\xi}{2}\right)-2\right],
$$

where $C$ and $D$ are constants, provided $p=1 / 2$. Hence

$$
\mu(\xi)=C \xi+D\left[-\sqrt{\pi} \xi e^{-\xi^{2} / 2} \operatorname{erf}\left(\frac{\xi}{2}\right)-2 e^{-\xi^{2} / 4}\right] .
$$

This gives a full solution for $u(t, x)$ in the form

$$
u(t, x)=C x+D\left[-\sqrt{\pi} x e^{-x^{2} / 2 t} \operatorname{erf}\left(\frac{x}{2 \sqrt{t}}\right)-2 \sqrt{t} e^{-x^{2} / 4 t}\right] .
$$


Taking the spatial derivative of the solution $u$ given by (45)

$$
\begin{aligned}
& \frac{\partial u}{\partial x}(t, x) \\
&=C+D\left[\sqrt{\pi} e^{-x^{2} / 2 t} \operatorname{erf}\left(\frac{x}{2 \sqrt{t}}\right)\left(\frac{x^{2}}{t}-1\right)\right. \\
&\left.+\frac{x}{\sqrt{t}}\left(e^{-x^{2} / 4 t}-e^{-3 x^{2} / 4 t}\right)\right] .
\end{aligned}
$$

From the Neumann boundary condition at $x=0$, we obtain

$$
\frac{\partial u}{\partial x}(t, 0)=C .
$$

It follows that $h(t)=q$ must be a constant. Hence

$$
C=-q,
$$

and from the Dirichlet condition we get

$$
-q s+D\left[-\sqrt{\pi} s e^{-s^{2} / 2 t} \operatorname{erf}\left(\frac{s}{2 \sqrt{t}}\right)-2 \sqrt{t} e^{-s^{2} / 4 t}\right]=u^{*}(t) .
$$

Let $\lambda=s / 2 \sqrt{t}$, and thus (49) becomes

$$
-2 q \lambda \sqrt{t}+D\left[-2 \sqrt{\pi} \lambda e^{-2 \lambda^{2}} \operatorname{erf}(\lambda)-2 e^{-\lambda^{2}}\right] \sqrt{t}=u^{*}(t) .
$$

The analytical solution based on a similarity variable [1] requires that $u^{*}=0$. This means that $u^{*}$ does not depend on time. In this treatment we will assume that $u^{*}$ is a function of $t$, that is, $u^{*}(t)=\alpha \sqrt{t}$, where $\alpha$ is a constant, which needs to be specified to realize an explicit solution. Thus

$$
-2 q \lambda+D\left[-2 \sqrt{\pi} \lambda e^{-2 \lambda^{2}} \operatorname{erf}(\lambda)-2 e^{-\lambda^{2}}\right]=\alpha .
$$

Since $D$ in (51) is a constant, it follows that $\lambda$ must also be constant. Thus

$$
\begin{gathered}
D=\frac{\alpha+2 q \lambda}{-2 \sqrt{\pi} \lambda e^{-2 \lambda^{2}} \operatorname{erf}(\lambda)-2 e^{-\lambda^{2}}}, \\
s(t)=2 \lambda \sqrt{t} .
\end{gathered}
$$

The Stefan condition at the free boundary $x=s(t)$ is

$$
s \frac{d s}{d t}=-\frac{\partial u}{\partial x}(t, s),
$$

where

$$
\begin{gathered}
s \frac{d s}{d t}=2 \lambda^{2} \\
\frac{\partial u}{\partial x}(t, s)=-q+D\left[\sqrt{\pi} e^{-2 \lambda^{2}} \operatorname{erf}(\lambda)\left(4 \lambda^{2}-1\right)\right. \\
\left.+2 \lambda\left(e^{-\lambda^{2}}-e^{-3 \lambda^{2}}\right)\right] .
\end{gathered}
$$

By putting (53) and (54) in the Stefan condition, we get the following transcendental equation:

$$
2 \lambda^{2}+D\left[\sqrt{\pi} e^{-2 \lambda^{2}} \operatorname{erf}(\lambda)\left(4 \lambda^{2}-1\right)+2 \lambda\left(e^{-\lambda^{2}}-e^{-3 \lambda^{2}}\right)\right]=q .
$$

Thus we have proved the following theorem.

Theorem 2. For the Stefan problem with variable latent heat

$$
\begin{gathered}
\frac{\partial u}{\partial t}=\frac{\partial^{2} u}{\partial x^{2}}, \quad 0 \leq x \leq s(t), \\
\frac{\partial u}{\partial x}(t, 0)=-q, \quad t>0, \\
u(t, s(t))=\alpha \sqrt{t}, \quad t>0, \\
s \frac{d s}{d t}=-\frac{\partial u}{\partial x}(t, s(t)), \quad t>0 .
\end{gathered}
$$

The solution is given by

$$
\begin{gathered}
u(t, x) \\
=-q x+D\left[-\sqrt{\pi} x e^{-x^{2} / 2 t} \operatorname{erf}\left(\frac{x}{2 \sqrt{t}}\right)-2 \sqrt{t} e^{-x^{2} / 4 t}\right] \\
s(t)=2 \lambda \sqrt{t}
\end{gathered}
$$

where

$$
\begin{gathered}
D=\frac{\alpha+2 q \lambda}{-2 \sqrt{\pi} \lambda e^{-2 \lambda^{2}} \operatorname{erf}(\lambda)-2 e^{-\lambda^{2}}}, \\
q=2 \lambda^{2}+D\left[\sqrt{\pi} e^{-2 \lambda^{2}} \operatorname{erf}(\lambda)\left(4 \lambda^{2}-1\right)\right. \\
\left.+2 \lambda\left(e^{-\lambda^{2}}-e^{-3 \lambda^{2}}\right)\right] .
\end{gathered}
$$

\section{Conflict of Interests}

The author declares that there is no conflict of interests regarding the publication of this paper.

\section{References}

[1] V. R. Voller, J. B. Swenson, and C. Paola, "An analytical solution for a Stefan problem with variable latent heat," International Journal of Heat and Mass Transfer, vol. 47, no. 24, pp. 5387-5390, 2004.

[2] J. B. Swenson, V. R. Voller, C. Paola, G. Parker, and J. G. Marr, "Fluvio-deltaic sedimentation: a generalized Stefan problem," European Journal of Applied Mathematics, vol. 11, no. 5, pp. 433452, 2000.

[3] J. Crank, Free and moving boundary problems, The Clarendon Press, Oxford University Press, New York, 1987.

[4] G. Adomian, Nonlinear Stochastic Operator Equations, Academic Press, Orlando, Fla, USA, 1986.

[5] G. Adomian, Nonlinear Stochastic Systems Theory and Applications to Physics, Kluwer Academic, Dordrecht, The Netherlands, 1989. 
[6] G. Adomian, Solving Frontier Problems of Physics: The Decomposition Method, vol. 60 of Fundamental Theories of Physics, Kluwer Academic, Dordrecht, The Netherlands, 1994.

[7] G. Adomian and R. Rach, "Transformation of series," Applied Mathematics Letters, vol. 4, no. 4, pp. 69-71, 1991.

[8] R. Rach, G. Adomian, and R. E. Meyers, "A modified decomposition," Computers and Mathematics with Applications, vol. 23, no. 1, pp. 17-23, 1992.

[9] G. Adomian and R. Rach, "Inhomogeneous nonlinear partial differential equations with variable coefficients," Applied Mathematics Letters, vol. 5, no. 2, pp. 11-12, 1992.

[10] G. Adomian and R. Rach, "Nonlinear transformation of seriespart II," Computers and Mathematics with Applications, vol. 23, no. 10, pp. 79-83, 1992.

[11] G. Adomian and R. Rach, "Modified decomposition solution of nonlinear partial differential equations," Applied Mathematics Letters, vol. 5, no. 6, pp. 29-30, 1992.

[12] G. Adomian and R. Rach, "Modified decomposition solution of linear and nonlinear boundary-value problems," Nonlinear Analysis: Theory, Methods \& Applications, vol. 23, no. 5, pp. 615619, 1994.

[13] G. Adomian and R. Rach, "Analytic solution of nonlinear boundary value problems in several dimensions by decomposition," Journal of Mathematical Analysis and Applications, vol. 174, no. 1, pp. 118-137, 1993.

[14] G. Adomian, "Modification of the decomposition approach to the heat equation," Journal of Mathematical Analysis and Applications, vol. 124, no. 1, pp. 290-291, 1987.

[15] G. Adomian, "A new approach to the heat equation-an application of the decomposition method," Journal of Mathematical Analysis and Applications, vol. 113, no. 1, pp. 202-209, 1986.

[16] A. Wazwaz, Partial Differential Equations and Solitary Waves Theory, Higher Education Press, Beijing, China; Springer, Berlin, Germany, 2009.

[17] A. M. Wazwaz, "Equality of partial solutions in the decomposition method for partial differential equations," International Journal of Computer Mathematics, vol. 65, no. 3-4, pp. 293-308, 1997.

[18] A. Wazwaz, "A reliable modification of Adomian decomposition method," Applied Mathematics and Computation, vol. 102, no. 1, pp. 77-86, 1999.

[19] J. Duan and R. Rach, "A new modification of the Adomian decomposition method for solving boundary value problems for higher order nonlinear differential equations," Applied Mathematics and Computation, vol. 218, no. 8, pp. 4090-4118, 2011.

[20] J. Duan, R. Rach, A. Wazwaz, T. Chaolu, and Z. Wang, "A new modified Adomian decomposition method and its multistage form for solving nonlinear boundary value problems with Robin boundary conditions," Applied Mathematical Modelling, vol. 37, no. 20-21, pp. 8687-8708, 2013.

[21] R. Rach, "A convenient computational form for the Adomian polynomials," Journal of Mathematical Analysis and Applications, vol. 102, no. 2, pp. 415-419, 1984.

[22] J. Duan, "Convenient analytic recurrence algorithms for the Adomian polynomials," Applied Mathematics and Computation, vol. 217, no. 13, pp. 6337-6348, 2011.

[23] A. Wazwaz, "A new algorithm for calculating Adomian polynomials for nonlinear operators," Applied Mathematics and Computation, vol. 111, no. 1, pp. 53-69, 2000.
[24] L. Bougoffa, R. C. Rach, and A. Mennouni, "An approximate method for solving a class of weakly-singular Volterra integrodifferential equations," Applied Mathematics and Computation, vol. 217, no. 22, pp. 8907-8913, 2011.

[25] L. Bougoffa, R. C. Rach, and A. Mennouni, "A convenient technique for solving linear and nonlinear Abel integral equations by the Adomian decomposition method," Applied Mathematics and Computation, vol. 218, no. 5, pp. 1785-1793, 2011. 


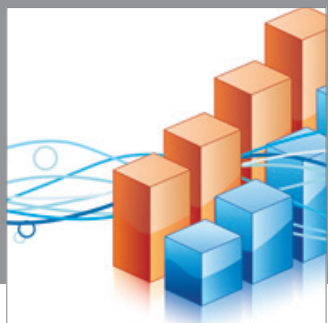

Advances in

Operations Research

mansans

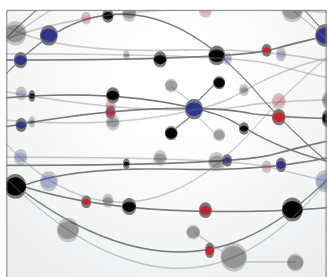

The Scientific World Journal
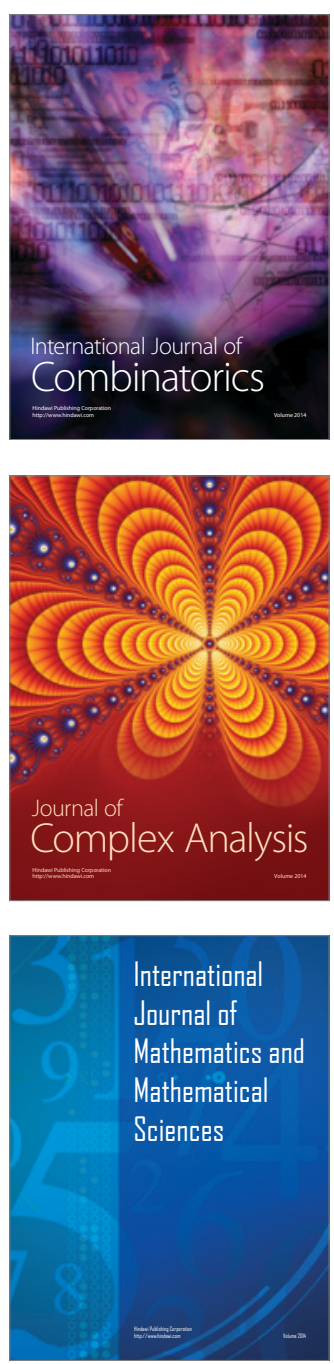
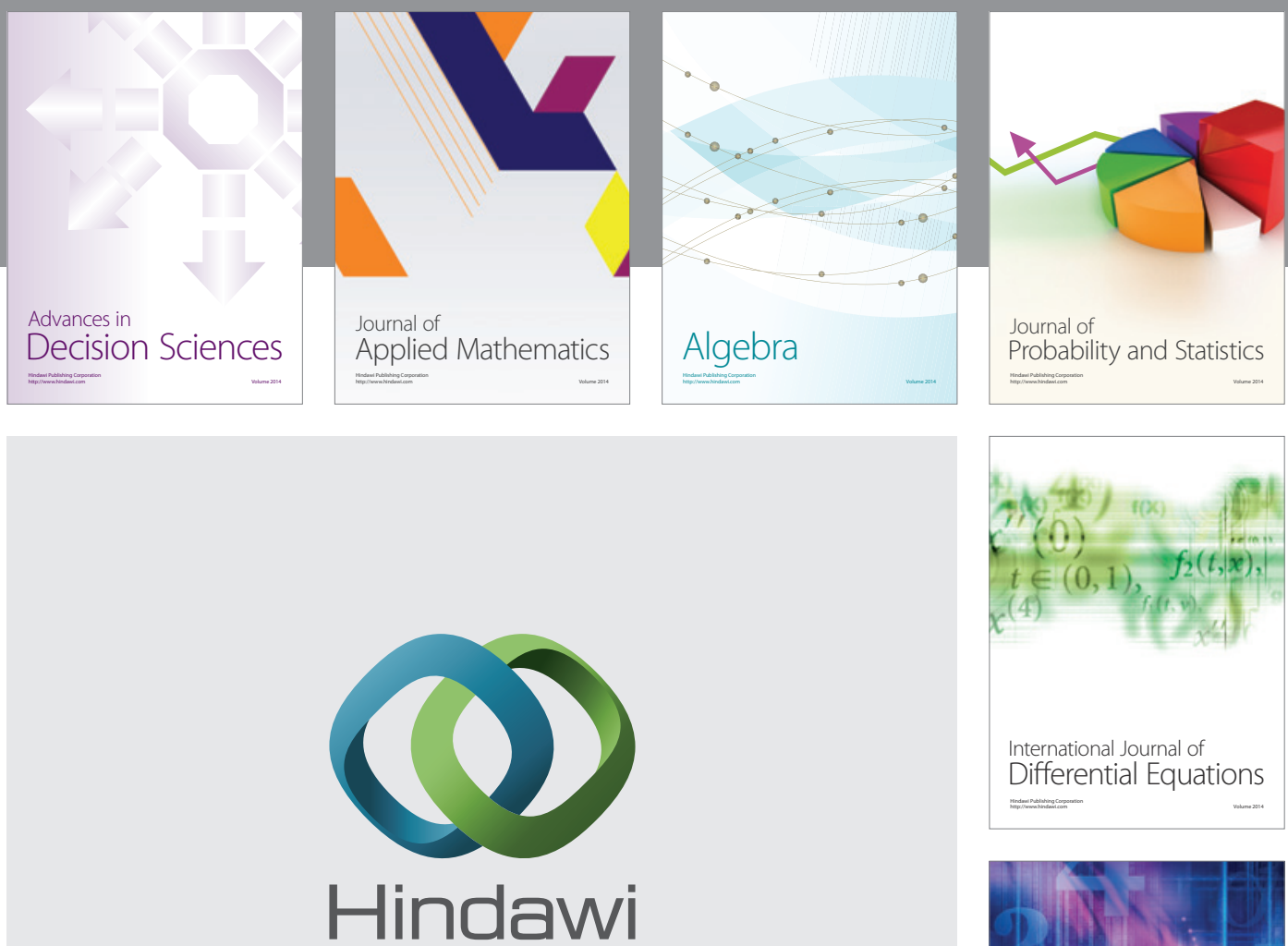

Submit your manuscripts at http://www.hindawi.com
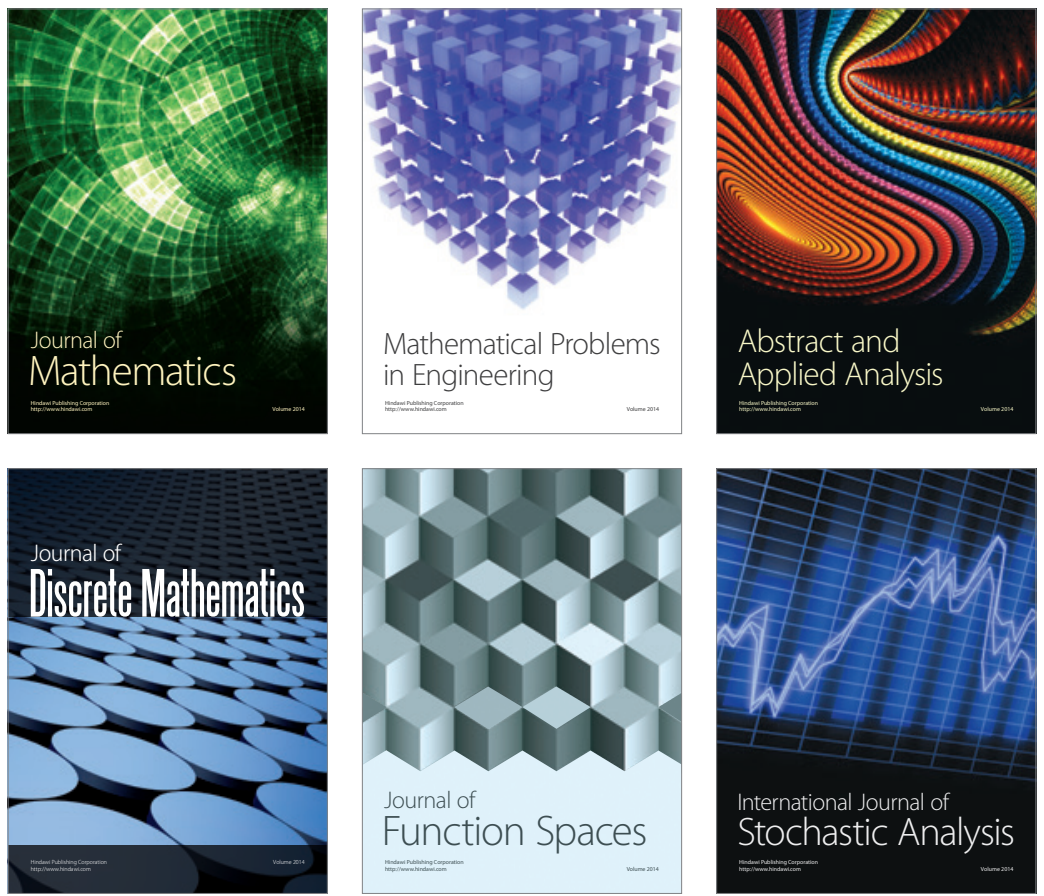

Journal of

Function Spaces

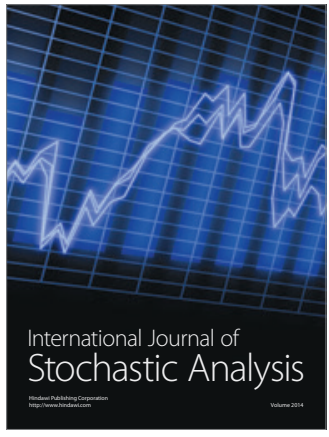

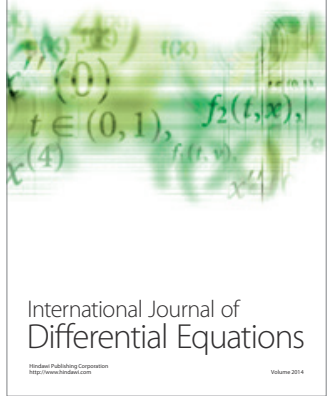
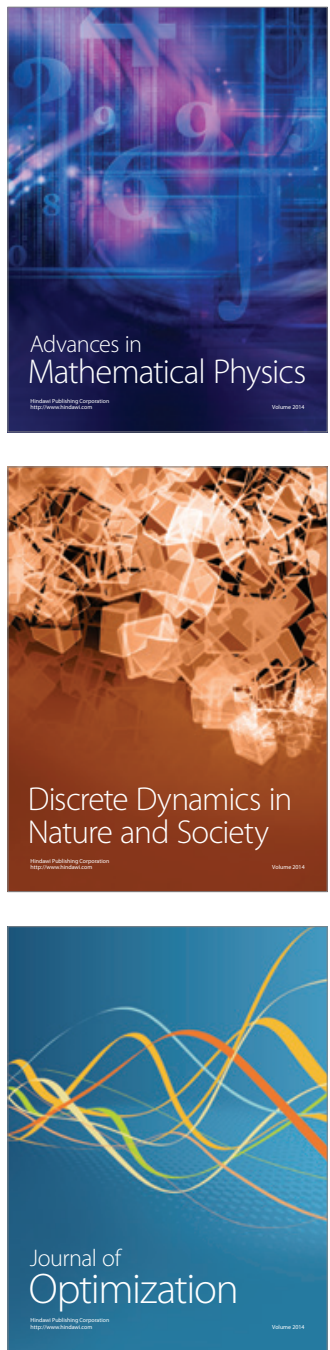